\title{
¿La experiencia Universitaria influye en el abandono de la Universidad?
}

\section{Does the University experience influence the abandonment of the University?}

\author{
Andrea Peña Fernández, MBA \\ Universidad Internacional del Ecuador, Ecuador
}

Autor para correspondencia: apena@uide.edu.ec

Fecha de recepción: 25 de Agosto de 2017 - Fecha de aceptación: 30 de Septiembre de 2017

\section{Resumen}

Se ha realizado un estudio exploratorio textual acerca de la deserción estudiantil a nivel universitario. El abandono de los programas académicos es una preocupación permanente, y a su vez esta se ve relacionada por la experiencia vivencial que el estudiante obtiene. La experiencia universitaria tiene relación directa a la toma de decisiones y de continuidad en el sistema educativo. Se mencionan varios factores posibles relacionados a componentes personales, sociales, del entorno, entre otros. Se concluye con un detalle resumido de los principales aspectos que influyen directamente en la deserción académica a partir de la experiencia universitaria.

Palabras clave: académica; deserción universitaria; experiencia académica; estudiantes; abandono estudiantil

\begin{abstract}
An exploratory textual study has been carried out on student dropout at university level. The abandonment of the academic programs is a permanent concern, and in turn this is related by the experiential experience that the student obtains. The university experience is directly related to decision making and continuity in the education system. Several possible factors related to personal, social, environmental components, among others, are mentioned. It concludes with a summary of the main aspects that directly influence the academic desertion from the university experience.
\end{abstract}

Key words: academic; university desertion; academic experience; students; student dropout 


\section{Introducción}

El abandono universitario se encuentra presente en todas las instituciones de nivel superior la misma que puede ser temporal o definitiva por parte de los estudiantes. Dicha deserción es ocasionada por las diferentes variables involucradas, siendo económico, vocacional, personal, factores internos o externos, entre otros. Existe potencialmente un gran número de factores que pueden tener causalidad en la cantidad de tiempo que lleva a los estudiantes abandonar sus estudios universitarios. Algunos de estos factores, pueden ser más fáciles de medir que otros, como el nivel de motivación del estudiante para estudiar, el nivel de integración académica y el tipo de condiciones de vida que existen en la universidad donde quieren estudiar.

El término "deserción universitaria" se utiliza comúnmente para describir situaciones en las que el estudiante abandona el estudio universitario en el programa que se inscribió antes de haber obtenido un título formal. Los términos utilizados para describir la deserción universitaria desde la perspectiva de los estudiantes son muchos: abandono, retiro, fracaso académico, falta de continuidad, no realización, mientras que sus contrapartes positivas son: persistencia, continuidad, graduación, etc., la tasa de deserción estudiantil es un término comúnmente aplicado, mientras que la retención y la tasa de graduación a menudo indican los resultados positivos.

El abandono universitario puede ser más o menos voluntario en el carácter como se ve desde la perspectiva individual del estudiante. Un estudiante puede abandonar su estudio universitario debido al incumplimiento de los estándares académicos y las exigencias dentro de la universidad, en cuyo caso la deserción debe ser vista como involuntaria. Por otro lado, un estudiante puede decidir abandonar su estudio universitario por razones de carácter más voluntario, por ejemplo, en busca de otra asignatura de estudio y/o de otra universidad, en cuyo caso el abandono se describe mejor como una transferencia de estudiante dentro del sistema de educación superior. Además, un estudiante puede abandonar el sistema de educación superior debido a circunstancias externas, por ejemplo, con respecto a dificultades financieras o problemas familiares o personales o, quizás, debido a un ciclo económico favorable. En el caso de dificultades financieras, problemas familiares o personales, aunque la decisión de irse haya sido tomada en parte por el alumno, la decisión no ha sido controlada por las autoridades universitarias y por lo tanto puede decirse que es más voluntaria en el carácter después de todo.

Como se muestra en este estudio, la distinción entre los diferentes tipos de abandono se la hace a partir de la experiencia estudiantil dentro de la universidad y debe tomarse muy en serio ya que a menudo son el resultado de diferentes procesos precedentes que se siguen configurando dentro de la universidad o que el estudiante no toma en cuenta. Un caso específico de abandono universitario también puede caracterizarse en el nivel institucional en el que se produce, ya sea de un curso o tema de estudio, un departamento, una facultad, una universidad o todo el sistema de educación superior. La deserción depende además de la perspectiva a partir de la cual se evalúa.

\section{Metodología}


Para la elaboración del presente artículo se realizó una búsqueda exploratoria a través de bibliografías consecuentes con el tema y se extrajo información de autores que tratan temas puntuales de deserción en experiencias universitaria tales como: Tinto, Castaño, Giraldo, Sánchez, Escobar, Fanelli, Ruiz, entre otros. Por otro lado se investigó acerca de estudios de diferentes universidades las mismas que analizan los diferentes factores negavitos que ocasiona la desercion y sus consecuencias. Finalmente se ha relacionado este estudio a la forma cómo la experiencia universitaria influye a los estudiantes durante su vida institucional y la relación con los diferentes actores de la comunidad universitaria.

\section{Desarrollo y Discusión}

La educación formal fue creada en múltiples divisiones es decir en ciclos, niveles y grados. Teniendo en cuenta que los estudiantes pueden elegir la institución de preferencia donde van a cruzar sus estudios, pero no son los únicos actores que están involucrados en ella. Otro actor involucrado son los educadores, ellos también pueden escoger la institución donde quieren impartir sus conocimientos. Dichos actores mencionados posteriormente, no pueden escogerse o seleccionarse entre sí, es decir quiénes van a estar en el aula, por lo que todos deben llevar diferentes roles con responsabilidad. Las instituciones interiormente poseen políticas y cada una desarrolla un estilo diferente de comunidad universitaria. La experiencia de vida y el nivel de abandono universitario constituyen uno de los itinerarios sobre la capacidad de gestión universitaria, tomando en cuenta la complejidad del sistema educativo y las altas exigencias sobre el cuidado de los bienes públicos, por lo que se requiere de una estructura organizacional de gestión ágil, oportuna, eficiente y eficaz (Giraldo, Abad, \& Díaz, 2005).

La experiencia universitaria, incide directamente en los estudiantes al menos en los primeros niveles de los programas educativos. El convivir diario trae nuevas experiencias a las que el estudiante debe acercarse y adaptarse, no solo en la parte social sino también en la parte política asumiendo deberes y derechos adquiridos interiormente. La universidad no es una tarea fácil, y muchos estudiantes descubren la manera de volverla difícil. Hay muchos factores que contribuyen a la falta de éxito. Algunos son inevitables; algunos son simplemente irresponsabilidad. De cualquier manera, el abandono de la universidad puede contar como una pérdida sustancial en la vida de un estudiante. Varios factores pueden afectar directamente en las actitudes y toma de decisiones de los estudiantes obligándoles a desertar temporal o definitivamente la educación superior. Entre estos casos relacionados a la experiencia estudiantil se puede mencionar:

\section{La expectativa del estudiante frente a la realidad y a la carga académica.}

Una vez que los estudiantes se integran completamente a su carga horaria en la universidad, se dan cuenta de que, a pesar de su nueva independencia, la cantidad de responsabilidades que viene con su libertad no era en absoluto lo que se anticipaba. Por lo tanto, se convierten en individuos desgastados y desalentados; por lo que renuncian, sin importar si sus calificaciones estaban bien o no. La exigencia académica se vincula directamente con la deserción (El Telégrafo, 2016), y muchas veces esto no depende de la madurez del estudiante sino que se atribuye al exceso de exigencias, de materiales bibliográficos contenidos en la materia, y de horas que insume llevar la materia al día (Universidad Nacional del Rosario, 2012). 
Es así que algunos estudiantes necesitan tiempo para ajustarse al mundo diverso de la universidad y desarrollar la variedad de habilidades apropiadas para afrontar los problemas derivados de las relaciones sociales en la comunidad universitaria (Tinto, 1989).

\section{Situaciones de vida y otras instancias externas}

Desafortunadamente, como la vida puede dictar, situaciones de vida inesperadas pueden ocurrir, y muchas veces son de una naturaleza tan grave que el estudiante se ve obligado a volver a casa. Una enfermedad o calamidad doméstica en la familia, las limitaciones financieras pueden contribuir a que un estudiante pueda estar obligado a renunciar a su educación universitaria por el momento o definitivamente. Refiriéndose a las enfermedades del estudiante o salud psicosomática, estas se forman por problemas de salud y situaciones emocionales y llevan al estudiante a sumirse en aspectos personales y terminan perjudicándolo en su desarrollo escolar. De ahí surge la necesidad de una ayuda psicológica e incluso siquiátrica que muchas de las veces la universidad no asume o talvez no se da por enterada (Sánchez, Navarro, \& García, 2009). Lamentablemente el estudiante asume deberes y derechos como se mencionó anteriormente y la inasistencia también deriva en la pérdida de nivel y si es reiterado en algunos casos la separación del alumno del programa de estudios acorde a la normativa institucional,

\section{Vida social}

El equilibrio de una vida social y académica es algo difícil para un estudiante universitario. Las amistades y el cambio de rutina pueden llevar a la degradación del estudiante que lo sigue y de quien lo conduce. La inclinación natural, por supuesto, es hacer amigos, disfrutar y el placer. Lo hábitos de beber, quedarse despierto hasta altas horas de la noche (y mañana), faltando a varias horas de sus clases hasta el punto en que el estudiante está demasiado lejos para compensar el espacio que exige la carrera universitaria puede incluso conducir a la expulsión, la cancelación del flujo de dinero de los padres o una decisión del propio estudiante de suspender la universidad. La experiencia de vida y la socialización en la Universidad, "no solo es un asunto del individuo que se pregunta por su identidad, sino que además se incluye el contexto social más amplio que posibilita u obstaculiza la consolidación de una identidad, donde será posible la coherencia de lo que se es, como persona, con lo que se quiere llegar a ser, en lo personal y profesional". (Escobar, 2013)

\section{Relaciones}

La interacción social a veces conlleva a encontrar el amor en la universidad siendo a veces esto muy común, pero cuando el amor se convierte en odio y angustia, puede fácilmente llevar al estudiante (o estudiantes) lejos de sus estudios académicos. Se convierte fácilmente en un pasajero sin rumbo, las clases compartidas, el mismo grupo de amigos, los dolorosos recuerdos, puede ser suficiente para que al menos uno de los dos tome la resolución de abandonar el programa académico. Muchas veces el casarse y unirse también deriva en la salida de la universidad (De la Peña, 2014). Ciertamente en estos casos la ayuda profesional y la convicción de las metas pueden contribuir a sobrellevar estos problemas y superarlos.

\section{Nostalgia}


Las comodidades del hogar siempre parecen ser deseables cuando un estudiante se encuentra solitario y desalentado. Ya se trate de una relación de abandono en casa o la comodidad de un dormitorio de niñez, su antiguo entorno o incluso el clima, para algunos estudiantes pueden llegar a ser tan abrumador por el dolor, y no pueden continuar en sus estudios. Según Páez (2011), el cambio de lugar de estudio o la transición a un nuevo estilo estudiantil universitario pueden causar cambios y producir en el sujeto un periodo de inadaptación a su nueva vida, consiguiendo llegar a momentos de aislamiento o baja motivación (Páez, 2011), en tal caso el estudiante debe tener la fortaleza de adaptarse a su nuevo entorno.

\section{Trabajo y estudios}

Algunos estudiantes que han conseguido un puesto de trabajo, por cualquier circunstancia, llegan a la conclusión de que ganar dinero es de alguna manera mejor que invertir su tiempo en una educación para posiblemente generar más dinero en el futuro, y renunciar a su carrera universitaria para centrarse en su trabajo. Refiriéndose al factor laboral, según Aguilera (2013), la mayoría de los estudiantes desertores afirma que se encontraba trabajando al momento de la deserción. Los alumnos por lo general no están trabajando al momento que realizan su ingreso a la facultad o programa universitario. En cuanto a la disponibilidad de tiempo para estudiar en relación al trabajo, la mayoría manifiesta que lo hacía por necesidad o porque su horario de estudios le permitía combinar las dos actividades, también aseveraron que casi siempre tenían limitaciones de horario para asistir regularmente a las clases debido al trabajo luego de haber tomado esa decisión (Aguilera \& Jiménez, 2013). La falta de recursos no se puede considerar una experiencia universitaria puesto que no es una vivencia adquirida dentro del campus universitario, pero la búsqueda de un trabajo sin que influya este factor si lo es.

\section{Ninguna atención u orientación vocacional}

El ser humano necesita una guía desde que va creciendo, se vive en medio de la dirección; prosperamos en la estructura y en la instrucción o cuidado de alguien. En la universidad, especialmente el primer año, la orientación y la atención individual de un consejero o mentor pueden no existir en la vida del estudiante. No saber a dónde ir, cómo lidiar con los problemas puede conducir a la mayoría de los factores que se menciona anteriormente $y$, finalmente, en el camino a una fallida experiencia universitaria. Una gran cantidad de estudiantes no tienen una visión clara si realmente tienen vocación por lo que estudian. A veces es un salto al vacío e ingresan a la universidad confiados en que realmente disfrutarán lo que han elegido o que a su vez en el transcurso puedan aceptar la carrera elegida; pero luego sufren una profunda decepción al encontrar que lo que estudian no es lo que esperaban (LogrosPerú.com, 2017).

Algunos estudiantes no están preparados para los programas académicos rigurosos necesarios para tener éxito en la universidad. Si la educación secundaria impuso poco o ningún esfuerzo de estudio, el estudiante tendrá un golpe muy fuerte y deberá acomodarse a las exigencias una vez que llegue a la universidad. Es cierto que la educación es un derecho universal como menciona Noro (2016), "La educación es un derecho fundamental para ejercer los otros derechos", la misma promueve la libertad y forja importantes beneficios para el desarrollo personal y desenvolvimiento dentro de la comunidad. Pero esto no significa que 
muchos estudiantes por su experiencia renuncien a su derecho y abandonen los programas estudiantiles.

Las distintas experiencias estudiantiles, atraviesan por "distintas esferas de la vida social (familiar, generacional, educativa, política, etcétera) que pone en juego horizontes diversos y contradictorios que se dirimen en buena medida en los procesos, dinámicas individuales y colectivas de la institución universitaria" (Carli, 2007)). Por ejemplo, en el ámbito educativo, si existe motivación entre los estudiantes por el aprendizaje asimilado o simplemente se encuentra el significado del porque se localizan en ese determinado espacio y tiempo, ocurrirá que ellos se vuelvan dinámicos al momento de alcanzar sus metas y objetivos trazados, siendo este un factor fundamental para evitar que ellos deserten sus estudios. Los agentes involucrados en el tema de deserción universitaria se componen de todos los individuos, políticas y procesos que componen el sistema educativo. "Este hecho, sin duda involucra directamente a todos los agentes del sistema educativo (Tinto, 2004). Generalmente el abandono de los programas académicos ocurre en los primeros semestres.

A pesar de los esfuerzos a través de múltiples investigaciones, inclusión de nuevos procesos y recomendaciones, la deserción dista de ser resuelta (De Vries, León, Romero, \& Hernández, 2011). Según Tinto (1989), en el caso de Estados Unidos de América, la tasa de deserción se ha mantenido constante en todo el siglo XX, a pesar de drásticos cambios en el sistema educativo y en el número de estudiantes (Tinto, 1989).

\section{Conclusión}

La deserción a partir de la experiencia universitaria, influye directamente en los estudiantes al menos en los primeros niveles de los programas educativos. La experiencia de vida y el nivel de abandono universitario constituyen uno de los problemas a resolver a través de la gestión universitaria, sin olvidar la complejidad del sistema educativo y las altas exigencias que se debe cumplir en un programa académico. Los casos sobre experiencia universitaria que desembocan en una deserción universitaria son varios entre los cuales se destacan: la expectativa académica relacionado a la carga horaria universitaria, los nuevos deberes que adquiere en la universidad, el ajuste necesario para adaptarse a la comunidad universitaria; las situaciones de vida y aspectos externos; la Vida social del estudiante, las Relaciones e interacción social, la nostalgia o condiciones que depriman al estudiante, el ingreso del estudiante a la fuerza de trabajo, la poca, nula, o deficiente orientación vocacional.

Los diferentes sujetos y entes involucrados en el tema de deserción universitaria son complejos, y se componen de todos los individuos, órganos, políticas y procesos que componen el sistema educativo de tercer nivel. Inclusive las nuevas políticas a nivel local procuran mejorar las tasas de retención estudiantil, tarea que no ha sido fácil en los últimos años. El avance en el estudio de la deserción universitaria relacionada a la experiencia estudiantil es complejo, y dependerá de la locación y la realidad del entorno, puesto que existen diferentes deficiencias y problemas en cada país y región. Esa misma complejidad ha hecho que este tipo de deserción haya podido eliminarse o disminuir los porcentajes por varios años.

\section{Bibliografía}


Aguilera, M., \& Jiménez, V. (2013). Factores De Deserción Universitaria En El Primer Curso De Las Carreras De Trabajo Social Y Lengua Inglesa En Las Facultades De Humanidades Y Ciencias De La Educación Y De Lenguas Vivas De La Universidad Evangélica Del Paraguay . Rev. Internacional Investigación Ciencias Sociales, 197-205.

Carli, S. (2007). La Experiencia Universitaria Y Las Narrativas Estudiantiles. Una Investigación Sobre El Tiempo Presente.

De La Peña, G. (2014). El Problema De La Deserción Escolar Y Las Posibilidades De La Investigación-Acción Participativa. Revista De Sociología Configuraciones, 129-149.

De Vries, W., León, P., Romero, J., \& Hernández, I. (2011). ¿Desertores O Decepcionados? Distintas Causas Para Abandonar Los Estudios Universitarios. Revista De La Educación Superior.

El Telégrafo. (11 De Noviembre De 2016). La Deserción Universitaria Tiene Cuatro Causas. Págs. $1-2$.

Escobar, J. (2013). Elección Profesional Y Deserción Universitaria. Psyconex, 1-17.

Giraldo, Abad, \& Díaz. (2005). Bases Para Una Política De Calidad De La Educación Superior En Colombia. Cna, 1-2.

Logrosperú.Com. (Enero De 2017). Deserción Universitaria Preocupa Al Mundo. Obtenido De Http://Www.Logrosperu.Com/Noticias/Actualidad/810-Desercion-UniversitariaPreocupa-Al-Mundo.Html

Noro, J. E. (2016). Sistema Educativo Y Universidad Planteos Históricos Y Debates Actuales. 1. Argentina: Universidad Nacional De Rosario.

Páez, R. (1 De Noviembre De 2011). Psicopedagogía Integral. Obtenido De Http://Psico3info.Blogspot.Com/2011/11/Desercion-Y-Sus-Principales-Factores.Html

Sánchez, G., Navarro, W., \& García, A. (2009). Factores De Deserción Estudiantil En La Universidad Sur colombiana. Paldela - Surcolombiana, 97-103.

Tinto, V. (1989). Definir La Deserción: Una Cuestión De Perspectiva. Revista De La Educación Superior, 1-2.

Universidad Nacional Del Rosario. (16 De Abril De 2012). Deserción Universitaria: Causas Y Razones Del Abandono. Obtenido De Http://Www.Fcpolit.Unr.Edu.Ar/DesercionUniversitaria-Causas-Y-Razones-Del-Abandono/ 